\title{
Conjunctival flap revisited: is it only a temporary solution for difficult corneal diseases?
}

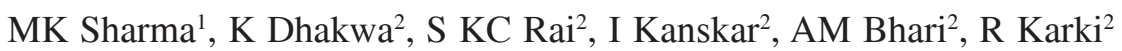 \\ ${ }^{1}$ BP Koirala Institute of Health Sciences, Dharan \\ ${ }^{2}$ Lumbini Eye Institute, Bhairahawa
}

\begin{abstract}
Background: The conjunctival flap technique, first described by Gundersen in 1958 has not undergone any major modifications since then, except for some variations depending upon pathology and surgeons' preferences. Objective: To show the importance of conjunctival (Gundersen) flap in different corneal diseases where keratoplasty is not possible immediately. Methods: Out of total 113 non-healing corneal ulcers of different etiologies, nine patients had undergone total conjunctival flap and the rest of the patients had partial conjunctival flap. Out of 104 partial flaps, 31 patients had bipedicle flap and 73 patients had pedicle conjunctival flap. Diagnoses for Gundersen flap surgery included chronic Non-healing infective keratitis with and without impending perforation(93) (fungal -30, no organisms on culture-42 and bacterial21), Neurotrophic keratitis(4),Herpes simplex keratitis(9),Bullous keratopathy(2),Infected nonhealing graft(2),presumed infective perforated keratitis(3)(anterior chamber formed after pad and bandage). Results: Eight flaps retracted due to ulcer necrosis out of which seven cases got perforated with complete healing of ulcer. One case had to be eviscerated due to panophthalmitis. One hundred five cases got cured. Conclusion: Conjunctival flap is the urgent and most reliable remedy (temporary or even permanent in some pathologies) for chronic nonresolving infective and presumed infective keratitis with or without impending perforation, neurotrophic keratitis, chronic herpes simplex keratitis, bullous keratopathy and persistent nonhealing epithelial defects where there are no immediate facilities for corneal transplantation. Conjunctival flap should be familiarized and emphasized as a primary and complementary surgical solution where penetrating keratoplasty or lamellar keratoplasty can't be immediately performed due to lack of corneal tissue.
\end{abstract}

Keywords: conjunctiva, surgical flap, epithelial transplantation, Gundersen flap, pedicle flap, bipedicle flap, corneal diseases, ocular surface diseases

\section{Introduction}

Conjunctival Flap is a technique described by Gundersen in the late 1950 for the treatment of chronic corneal ulcerations. This technique, first described by Gundersen in 1958 has not undergone any major modifications since then, except for some variations depending upon pathology and surgeons' preferences. The conjunctival flap acts as a biological patch having trophic, protective and analgesic effect. As a result of such effect, the aesthetic appearance of the eye improves and the corneal conditions for grafting, as well. ${ }^{5}$ No doubt; many chronic infective and non-

Address for correspondence:

Dr Manoj K. Sharma, Additional Professor

Department of Ophthalmology, BPKIHS, Dharan, Sunsari, Nepal Email: mansuyu@gmail.com infective ulcers heal faster and also maintain the integrity in cases of impending perforations. The conjunctival flap is well-proven, time-honored treatment for numerous desperate corneal diseases sharing in common, a persistently compromised ocular surface. Ocular surface is typically damaged because of neurotrophic or neuro-paralytic diseases, severe dry eyes, or bullous keratopathy. In doing so, the flap prevents progressive corneal ulceration and secondary infection, as well as controls pain, eliminate frequent medications, improve cosmesis, and sometimes provide alternative to invasive surgery or enucleation .2

A selected pedunculated conjunctival flap is an effective and practical surgical approach for the 
treatment of perforated and non -perforated corneal ulcers that have not responded to other medical therapy. ${ }^{3}$ The conjunctival flap can be performed totally or partially, and partial flaps are classified as bridge, hood (advancement of conjunctival flap) or racquet flap (pedicle flap). A 360 degree peritomy is performed in total flap whereas a 60 to 180 degree peritomy is made in partial flaps. ${ }^{5}$

A non-healing corneal ulcer is resistant to treatment, particularly in debilitating ocular surface. The ulcers may progress rapidly and may result in corneal perforations that lead to devastating visual sequelae. Restoration of the integrity of the globe and ocular tension is necessary to preserve the visual potential. The conjunctival flap such as Gundersen flaps and partial conjunctival (pedicle and Bipedicle) have been used fairly extensively in the management of nonhealing ulcers. ${ }^{2}$

It is a well proven for the numerous corneal diseases resistant to medical treatment. ${ }^{3}$ Conjunctival flap restores the integrity of chronically compromised corneal surface. It can also restore the integrity of the eye ball. ${ }^{4}$ It has a potential for limited visual improvement, too. Surgery is simple, not aggressive and with low cost, also with low complication rate. It has immediate and sustained benefit. Sometimes, destructive surgery like enucleation can also be avoided. The flap can also provide a surface for the placement of a cosmetic scleral shell or painted contact lens. ${ }^{1}$

Conjunctival flaps are indicated when medical treatment, including therapeutic contact lens has failed; the flaps are also indicated when tarsorraphy is insufficient and corneal grafting is considered risky and in cases of readily unavailable donor tissues. Restoration of the integrity of the globe, ocular surface and ocular tension is necessary to preserve the visual potential. $^{2}$

Cases like aphakic or pseudophakic keratopathy are sometimes very irritating and annoying to the patient .If the bullae get ruptured, they will be very painful and sometimes get infected, too. If cornea is not readily available for the transplant, the alternative treatment in those cases would be conjunctivoplasty. The patient may be planned to cover only that portion of the cornea where the edema is most intense, usually where the bullae occur. In cases, where the edema is generalized and bullae are present over the entire cornea, a complete conjunctival flap must be applied. ${ }^{10}$
In Lumbini Eye Institute, a large number of patients attend in OPD with corneal ulcer (infective and non infective) each year. For example, the number of perforated corneal ulcer attended in cornea clinic in 2005 was 232. Number of perforated corneal ulcer attended in cornea clinic from January to May 2006 was 47. Apart from these cases, total number of corneal ulcer scrapped for the identification of the organism was 1271 in the year 2005. Among them, 225 were bacterial ulcers, 508 fungal ulcers, 25 with multiple organisms and no organisms on culture were 509 cases.

\section{Conjunctival flap in LEI: A glance}

(October 2005 - May 2006)

\begin{tabular}{|l|c|c|}
\hline \multicolumn{1}{|c|}{ Disease } & $\begin{array}{c}\text { Number of } \\
\text { cases }\end{array}$ & \% \\
\hline Chronic infective corneal ulcer & 70 & 62 \\
\hline $\begin{array}{l}\text { Corneal ulcer with impending } \\
\text { perforation }\end{array}$ & 23 & 21 \\
\hline Chronic herpetic keratitis & 9 & 8 \\
\hline Neurotrophic keratitis & 4 & 4 \\
\hline Pseudophakic bullous keratopathy & 1 & 1 \\
\hline Aphakic bullous keratopathy & 1 & 1 \\
\hline Infected non healing corneal graft & 2 & 2 \\
\hline Infected perforated corneal ulcer & 3 & 3 \\
\hline
\end{tabular}

Most of the cases of corneal ulcers were of infective origin attending in the cornea clinic. A large number of them were recalcitrant to medical treatment as the cases were chronic. Many of them had used topical and also oral steroid and worsened the course of the disease. Patients had poor socioeconomic status, poor compliance to drugs and follow-up visits.

\section{Methods}

Under aseptic condition, all the microsurgical procedures were done in peribulbar anesthesia with 2 $\mathrm{ml}$ of $2 \%$ xylocaine with adrenaline . The eye is cleaned gently with $5 \%$ betadine.

Barraquer's wire speculums were not used to separate the lids and expose the ocular surface in the cases of impending perforations. The procedure is performed under the operating microscope.

Total conjunctival flaps were done for lesions extending nearly the whole cornea as in bullous keratopathy; some non-healing infective or presumed infective, nearly total or total corneal ulcers; in large corneal defects as in herpetic keratitis; neurotrophic keratitis. Traction suture with 6:0 silk was applied on the limbus in the supero-temporal or other areas depending upon 
Sharma MK et al

Health Renaissance, January-April 2012; Vol 10 (No. 1);40-45

Conjunctival flap revisited

the site of the lesion. Traction suture was not necessary for the Partial Gundersen flap(PGF).

In cases of chronic ulcers, conjunctival flap were done in cases not responding to medical treatment for more than 3 weeks, poor compliance of the patients and in impending perforations. Total conjunctival flaps were done by free graft technique obtaining free graft from the superotemporal area, but not exactly like classical Gundersen flap technique. Only epithelial debridement up to the periphery was done on the corneal bed before placement of the flap.

For partial conjunctival flap, pedicle flap was chosen for the non healing ulcers or for the impending perforations of the para- central or peripheral areas.

Debridement of the ulcer was done, removing necrotic tissue material and with lamellar keratectomy in few cases. $^{6}$ The epithelial debridement was done also around the ulcer site about $0.5 \mathrm{~mm}$ to $1 \mathrm{~mm}$ adjacent to the ulcer margin. The debridement of the epithelium was done nearly up to the limbal region starting from the ulcer site. The lesion and the adjacent area was treated with betadine $5 \%$ with the help of cotton tip applicator.

In cases of sterile cases, simply epithelial debridement up to the limbus was done.

For the non-healing chronic peripheral or central ulcers, pedicle or bipedicle flaps were taken. Flaps were taken from the nasal or temporal site, also from the superior or inferior site depending upon the location of the lesion. After approximating the width of the lesion, the conjunctival flap was made adjacent to the lesion, taking out ribbon or belt like conjunctival flap attached to its base. For this, with the help of the serrated forceps, the conjunctiva was grasped and button-hole was created. With the help of Westcott scissors, gradually closing and advancing its tips, blunt dissection of the tenon and episcleral tissues were done. Likewise, the button-hole was extended superiorly or inferiorly, medially or laterally parallel to the limbus. Approximately, the peritomy was done of the length parallel to it $\left(60^{\circ}\right.$ to $\left.180^{\circ}\right)$, thus creating a hanging pedicle or bipedicle flap. Then the flap was advanced towards the corneal lesion, approximated and sutured on the corneal bed anchoring anterior healthy corneal stroma with 10:0 nylon, with long cut suture tips in place so that it would not irritate in the post-op 2 weeks period and also easy for suture removal. Bipedicle flap was placed vertically. Sutures were applied on both the edges of the flap, in the distal end, in the middle of the flap and in the limbal region. In cases of TGF, interrupted sutures were applied $360^{\circ}$ all around the peritomized conjunctiva and free conjunctival flap. Underlying tenon was still undermined if traction was felt intraoperatively. Flap was secured not too tight or not too loose on the recipient bed. Accidental buttonhole in the flap was sutured immediately during surgery.

For the pedicle grafts, flaps were taken from the nearest area of the lesion from the limbus considering the better vascular supply. Conjunctiva along with some tenon's tissue were taken for the cases with thin cornea. For the non healing herpetic lesions, epithelial debridement about $0.5-1 \mathrm{~mm}$ from the border of whole lesion was done like in infective other cases.

For the infected, recently perforated (according to the history and clinical examination) 3 ulcer cases, firm pad and bandage was done for 24 hours, anterior chamber was formed and conjunctival flap surgery, pedicle flap, was done on the following days.

In 3 cases with impending perforations, which perforated during peribulbar anesthesia and in 1 case intraoperatively, anterior chamber was formed with air. Ulcer healed with formed AC.

Non-infective cases were treated with topical predisolone acetate $1 \%$ and ofloxacin post-operatively for six weeks.

Infective cases were treated with pre-op medications immediately after 1 hour of surgery.

Frequency of the drugs also reduced. All cases had got frequent lubricating agent simultaneously .

\section{Results}

Table 1: Corneal Diseases

\begin{tabular}{|l|c|c|}
\hline \multicolumn{1}{|c|}{ Corneal Diseases } & $\begin{array}{c}\text { No. of } \\
\text { cases }\end{array}$ & \% \\
\hline $\begin{array}{l}\text { Chronic non-healing infective/presumed } \\
\text { infective keratitis } \\
\text { with or without impending perforation }\end{array}$ & 93 & 82 \\
\hline Neurotrophic keratitis & 4 & 3 \\
\hline Herpex simplex keratitis & 9 & 8 \\
\hline Bullous keratopathy & 2 & 2 \\
\hline Infected non-healing graft & 2 & 2 \\
\hline Presumed infective keratitis (Perforated)* & 3 & 3 \\
\hline Total number of patients & $\mathbf{1 1 3}$ & $\mathbf{1 0 0}$ \\
\hline
\end{tabular}

*anterior chamber formed after pad and bandage overnight or intraoperatively after flap placement. 
Table 2: Flap type

\begin{tabular}{|c|c|c|}
\hline \multicolumn{1}{|c|}{ Flap type } & No. of cases & \% \\
\hline Total Gundersen flap & 9 & 8 \\
\hline Partial conjunctival flap & 104 & 92 \\
\hline Pedicle flap (73) & & \\
\hline Bipedicle flap (31) & & \\
\hline Total number of patients & $\mathbf{1 1 3}$ & $\mathbf{1 0 0}$ \\
\hline
\end{tabular}

Table 3: Treatment outcome

\begin{tabular}{|c|c|c|}
\hline & No. of cases & \% \\
\hline Totally or partially retracted flap & 8 & 7 \\
\hline $\begin{array}{c}\text { Perforated but healed ulcer } \\
\text { among the above cases (7) }\end{array}$ & & \\
\hline $\begin{array}{c}\text { Corneal perforation and } \\
\text { panophthalmitis (1) }\end{array}$ & & \\
\hline Successfully treated cases & 105 & 93 \\
\hline Total number of patients & $\mathbf{1 1 3}$ & $\mathbf{1 0 0}$ \\
\hline
\end{tabular}

Eight flaps retracted partially or completely due to ulcer necrosis. Out of which seven cases got perforated with complete healing of ulcer. One case had to be eviscerated due to panophthalmitis. One hundred five cases got cured.

All cases got relief from symptoms immediately after the surgery. Most cases got healed, and the ulcer resolved within 2 weeks on the $2^{\text {nd }}$ follow up visit. Suture removal was done at that time. Some flap got partially necrosed and partially retracted but the lesion was healed without perforation (Table 1, 2 and 3).

One case with neuroprophic keratopathy got flap retracted twice and had undergone lateral tarsorraphy.

Good vascularization of the flap was seen adjoining limbal vessels in the $2^{\text {nd }}$ follow-up visit after 2 weeks in cases of free and rotational total conjunctival flap grafts. Some visual recovery was there with absolutely no complaints in cases of mostly bullous keratopathy, chronic herpetic keratitis, neurotrophic keratitis.

One case of pseudophakic bullous keratopathy got intraocular pressure lowered.

Both the infected graft cases were healing in first follow-op visit. Only the integrity of the eye-ball could be saved.

Flap cases with hypopyon, corneal ulcer had secondary glaucoma and are still under anti-glaucoma drugs. Most of them had undergone hypopyon removal during the surgery. One case of one-eyed diabetic patient, with chronic herpetic keratitis for more than 15 years got
Sharma $M K$ et al Health Renaissance, January-April 2012; Vol 10 (No. 1);40-45 Conjunctival flap revisited

cured with 2 step surgery. Bipedicle flap surgery was followed y ECCE with PCIOL and optical iredectomy with some useful vision (P.L. to F.C. 1/60) to the patient.

None of the relapsed cases of herpes simplex keratitis, neurotrophic keratopathy was seen in the clinic during the period of about 2 and half years treated with conjunctival flaps.

Patients treated mostly with total conjunctival flaps complained no more symptoms. Even vision was improved in some cases; intraocular pressure was lowered in one case.

Many infective corneal ulcer patients with impending corneal perforation got cured rapidly, restoring the integrity of the eye ball and also future visual potential.

\section{Discussion}

A conjunctival flap help to maintain the integrity of the eye and prevent infection where penetrating or lamellar keratoplasty is not feasible or donor tissues and other resources are not available .A conjunctival flap may be placed to prepare the ocular surface for a cosmetic scleral shell that can erode the corneal epithelium. The epithelium of the conjunctiva is less subject to erosion than that of the cornea, and a conjunctival flap creates a surface more tolerant of the shell.

Corneal transplantation is not always suitable, an inflamed eye, highly vascularized cornea, for example, can have high risk of graft rejection. Coming to the use of tissue glue, it has also its drawbacks. It may result in protracted course of recovery and has limited use in the setting or prolapsed uveal tissue through corneal perforation. ${ }^{9}$

As already stated in the beginning, corneal ulcers caused by various micro-organisms that are resistant to medical therapy can be treated with a conjunctival flap. Although corneal stroma contains elements of the humoral immune systems(immunoglobins), it lacks elements of the cell-mediated immune systems( $\mathrm{T}$ cells).A conjunctival vessels can provide blood vessels and lymphatic channels that increase exposure of the invading organisms to immunologic defenses. Though there are reports of successful treatment of medically resistant bacterial keratitis or fungal keratitis or ulcers with no organisms in culture, keratitis should not be treated with a conjunctival flap unless the infected 
Sharma MK et al

Health Renaissance, January-April 2012; Vol 10 (No. 1);40-45

Conjunctival flap revisited

area has first been sterilized with antibiotics and is free of necrotic corneal tissue. Graft itself can be necrotized in such cases and surgery will not be beneficial. This author has himself experience of necrotized conjunctival flaps when the ulcers were not ready for the flaps. In this series, 6 cases had progressive corneal ulceration, sloughing of the corneal stroma along with conjunctival flap and corneal perforations. Advances in antifungal agents have made medical therapy the treatment of choice for most cases of fungal keratitis, however, the frequent use of topical, sub-conjunctival, and/or systemic antifungal agents for several weeks is generally necessary., some patients will not or cannot comply with such an intensive regimen. Even in patients who can comply, a lamellar keratectomy combined with a conjunctival flap is often an excellent alternative to medical therapy, especially if the infection is peripheral. There is usually time for a trial of medical therapy in the course of fungal keratitis, but lack of response or progression of the keratitis is an indication for surgical intervention if the infection is peripheral, a lamellar keratectomy is performed to remove all the necrotic material, and a partial conjunctival flap is placed on the keratectomy bed. $^{6}$

The same above mentioned authors argue for penetrating keratoplasty if the infection is central and lamellar keratoplasty and total conjunctival flap if the infection is more extensive and involve central and peripheral cornea. ${ }^{6}$ Our series of infective central ulcers with impending perforation shows healing of the ulcers with bipedicle flap in eight cases. Bipedicle flap was vertically placed to minimize the trauma to the flap by lid movements and less vulnerable to retract.

In cases of herpes simplex and herpes zoster viral keratitis, especially if there is metaherpetic keratitis, and in cases of frequent recurrent herpetic keratitis, there is dramatic healing of the lesion and the eye quiets faster. The recurrence rate is also low after the flap. Herpes Zosters and herpes simplex viral keratitis can cause recurrent and long-standing corneal inflammation, persistent non-healing epithelial defects, and stroma ulceration. Medical therapy consisting of antiviral, corticosteroids, and ocular lubricates and bandage soft contact lenses are effective in most patients. Prolonged morbidity is common, especially if the eye is dry and desensitized. Frequent instillation of topical medications and multiple visits in the cornea clinic often become more burdensome to the patient than the keratitis itself.

A conjunctival flap can help a patient whose life is dominated by the keratitis; patient becomes more comfortable without the need for constant medical attention. A lamellar keratectomy to remove necrotic cornea followed by a conjunctival flap can relieve inflammation, pain and other symptoms in as little as one week. The flap shortens the recovery time, and the penetrating keratoplasty can be postponed. The keratoplasty result in quiet eyes is better than in eyes with active lesions. ${ }^{9}$ The recurrence rate is also low after the flap placement. Some got visual improvement by even 2,3 lines in Snellen's chart even with the flap in situ. This is possibly because of halted inflammation after the flap placement and thinned out flaps after several months or year. In this case series study, there were 23 cases of corneal ulcer with impending perforation. There were 3 cases with history of recently perforated cornea and with 3 cases which perforated intraoperatively. Among the 23 cases, 7 cases got failed in restoring the integrity of the eye ball. All 6 perforated cases did well. So, among the 113 cases, 105 cases got cured. In this study, the criteria of successful treatment were-immediate relief of pain, no analgesics during sleep or reduced, sealed perforation or restored AC even after 14 days, infection clinically controlled, and scar formation. The frequency of drugs were also reduced. A selective pedunculated conjunctival flap is an effective and practical surgical approach in the treatment of perforated and nonperforated corneal ulcers that have not responded to other medical therapy. ${ }^{3}$

Although the construction of a conjunctival flap is relatively straight forward, it has some intra operative and post operative complications. Retraction is due to tension on the flap and if is noticed postoperatively, it is possible to undermine the flap to release the tension and flap can be sutured back into the place. Another complication is button-holing of the flap. This study shows that small button-holing do not effect the outcome if there is no tension on the well placed flap. But if the button hole is large, they may enlarge and destroy the flap. It should be repaired by undermining the edges of the hole, removing any epithelium that has grown onto the cornea through the hole, and suture the edges of the hole to each other and to the underlying cornea. Epithelial cysts are another 
complication which is troubleshooting. They are seen when retained epithelium proliferate under the conjunctival flap and can separate the cornea from the conjunctival flap. In this study, we have seen epithelial cysts growing after 15 days at the time of suture removal in 2 pedicle flaps of non healing ulcer .Ulcer was about to heal by that time and the flap was removed with the cysts after one month of the surgery when the ulcer healed.

Sub-conjunctival hemorrhage, ptosis, symblepharon are other complications which can be intra-operative or post-operative. One case of aphakic and one case of pseudophakic bullous keratopathy had undergone total conjunctival flap(TCF).The technique was basically Gundersen's technique modified by Juan Anesten. ${ }^{7}$ Anne Marie Alino(conjunctival flaps) In our study, for the TCF ,the flap was taken from supero-temporal area, but there was no symblepharon formation or ptosis in the late post-op period. The patients were asymptomatic after many years of irritation, photophobia and watering and there was visual improvement from F.C.close to face to $1 / 60$ in aphakic and from $6 / 60$ to $6 / 24$ in pseudophakic patient. So, thin flap from supero-temporal conjunctiva may also be ideal for TCF.

\section{Conclusion}

Conjunctival flap is the urgent and most reliable remedy (temporary or permanent) for chronic nonresolving infective and presumed infective keratitis with or without impending perforation, neurotrophic keratitis, chronic herpes simplex keratitis, bullous keratopathy and persistent non-healing epithelial defects where there are no immediate facilities for corneal transplantation. Conjunctival flap should be familiarized and emphasized as a primary and complementary surgical solution where penetrating keratoplasty or lamellar keratoplasty can't be immediately performed due to lack of corneal tissue.

\section{Acknowledgement}

We would like to thank Mr. Pawan Tiwari, Technician, Microlab, LEI and OT assistants for their help for this study. We are obliged to Mrs. Sudha Rishal Sharma, Librarian, and Mr. Amit Dahal, Library Assistant and highly appreciate their help for searching the literature as well as assisting in preparing this article.

\section{References}

1. Alino AM, Perry HD, Kanellopoulos AJ, Donnenfeld, ED. Conjunctival flaps. Ophthalmology 1998;105(60):1120-23.

2. Sandinha T, Zaher SS, Roberts F, Devlin HC, Dhillon B, Ramesh K. Superior fornicial conjunctival advancement pedicles (SFCAP) in the management of acute and impending corneal perforations. Eye 2006;20: 84-9.

3. Khodadoust Ali, Quinter AP. Microsurgical approach to the conjunctival flap. Arch Ophthalmol. 2003;121:1189-93.

4. Gundersen Trygue, Pearlson, HR. Conjunctival flaps for corneal disease: their usefulness and complications. Trans Am Ophthalmol Soc. 1969;67:78-95.

5. Geria RC, Zarate J, Marcos G. Penetrating keratoplasty in eyes treated with conjunctival flaps. Cornea 2001;20:345-49.

6. Kaufman, HE. Barron BA, McDonald MB. The Cornea: $2^{\text {nd }} e d$. Boston: Buttterworth; 1998: 727734.

7. Arentsen, JS. The use of conjunctival free grafts on the cornea. In : King, JH editor- The Cornea World Congress; 1964: Washington DC: October; Washington: Butterworts; 1965. 564-569.

8. Lauring L, Wergeland FL. Total Conjunctival Flap with a modification of the Gundersen Operation. Am J Ophthalmol. 1973; 76(6): 953-956.

9. Roberto CG, Richardo D, Wainsztein MD, Mario B, Richardo B, Marcos A . Infectious keratitis in the corneal grafts: treatment with partial conjunctival flaps. Ophthalmic Surg Lasers and Imaging. 2005: 36(4):298-302.

10. Gundersen, T. Conjuctival flaps in the treatment of corneal diseases with reference to a new technique of application. Archs Ophthal. 1958; 60: 880-887. 